\title{
Intrinsic photoluminescence of adamantane in the ultraviolet spectral region
}

\author{
Lasse Landt, ${ }^{1}$ Witoslaw Kielich, ${ }^{2}$ David Wolter, ${ }^{1}$ Matthias Staiger, ${ }^{1}$ Arno Ehresmann, ${ }^{2}$ Thomas Möller, ${ }^{1}$ and \\ Christoph Bostedt ${ }^{1}$ \\ ${ }^{1}$ Institut für Optik und Atomare Physik, Technische Universität Berlin, Eugene-Wigner-Bldg. EW 3-1, \\ Hardenbergstr. 36, 10623 Berlin, Germany \\ ${ }^{2}$ Institute of Physics, University of Kassel, 34132 Kassel, Germany
}

(Received 20 August 2009; published 24 November 2009)

\begin{abstract}
We report intrinsic photoluminescence in the ultraviolet for adamantane $\left(\mathrm{C}_{10} \mathrm{H}_{16}\right)$, the smallest in a series of hydrogen-passivated diamond clusters (diamondoids). The luminescence is ascribed to recombination of selftrapped excitons. The inclusion of high amounts of nitrogen into the nanodiamond's crystal lattice, using the example of urotropine (hexamethylenetetramine), is found to quench the luminescence. The results show that diamondoids are promising semiconductor nanocrystals for nanophotonic applications in the ultraviolet spectral region.
\end{abstract}

DOI: 10.1103/PhysRevB.80.205323

PACS number(s): 78.67.Bf, 78.55.Ap, 33.20.Ni, 73.22.-f

\section{INTRODUCTION}

Semiconductor nanocrystals (NCs) have attracted considerable attention due to their size-dependent electronic structure. ${ }^{1,2}$ While large parts of the semiconductor industry are silicon-based, light-emitting devices are in general made from direct band gap compound semiconductors, such as GaAs. The discovery of luminescence from Si nanostructures in 1990 (Ref. 3) sparked interest in the light emission properties of elemental indirect band gap semiconductor nanostructures ${ }^{4,5}$ and revealed new possibilities to implement light-emitting devices. Even though there has been considerable progress in the understanding of Si luminescence, the details of its origins are still a matter of debate. ${ }^{4}$ Quantum confinement effects and the breakdown of the momentum conservation in nanostructures, ${ }^{6}$ as well as highly localized defect states, ${ }^{4}$ play a decisive role. Despite the majority of work focused on $\mathrm{Si}$, the underlying concepts are expected to be of universal nature. However, it is still unclear if and how these concepts extend to indirect band gap semiconductors other than $\mathrm{Si}$.

In this paper, we report on intrinsic photoluminescence (PL) of the smallest diamond nanostructure, adamantane $\left(\mathrm{C}_{10} \mathrm{H}_{16}\right)$. We find that this diamond molecule of $0.5 \mathrm{~nm}$ diameter $(0.3 \mathrm{~nm} \text { for the carbon cage })^{7}$ exhibits a broad ultraviolet (UV) luminescence when photoexcited above its principal gap of $6.49 \mathrm{eV}^{8} \mathrm{UV}$ luminescence is found to be quenched by introducing nitrogen into the adamantane lattice.

Adamantane $\left(\mathrm{C}_{10} \mathrm{H}_{16}\right)$, shown in Fig. 1(b), is a hydrogenpassivated and fully $\mathrm{sp}^{3}$-hybridized carbon cluster. It is the smallest closed-cage structure in bulk diamond configuration consisting of only a single crystal cage. As such, it is the starting point of a series of perfectly defined diamond NCs, called diamondoids. ${ }^{9}$ Diamondoids exhibit a variety of interesting electronic properties, ${ }^{10-12}$ as well as a size- and shapedependent optical absorption in the UV. ${ }^{8}$ Furthermore, they can be introduced into solid state devices via self-assembled monolayers ${ }^{13,14}$ and they are biocompatible, making them attractive for applications in nanotechnology.

\section{EXPERIMENT}

The adamantane samples investigated in this work exhibit purities exceeding 99\% and have been obtained from SigmaAldrich. To avoid interaction of the NCs with their surroundings as well as among each other, experiments were performed in the gas phase. ${ }^{15}$ For investigation of the PL, we employ synchrotron radiation from beamline U125/2 at the Berlin synchrotron facility BESSY II. The incoming photon beam is monochromatized using a 10 -m normal-incidence monochromator equipped with a $300 \mathrm{l} / \mathrm{mm}$ grating yielding a spectral resolution better than $30 \mathrm{meV}$. To confine the interaction of the synchrotron beam with the sample to a small well-defined volume, an absorption cell setup is used, which is placed inside a vacuum chamber. The absorption cell uses three $\mathrm{MgF}_{2}$ windows, two in line with the synchrotron beam to measure transmission and one perpendicular to the incident beam to allow the detection of luminescence. The light transmitted through the absorption cell is a measurement for the absorption ${ }^{8}$ and is used to estimate the quantum yield (QY). The PL is dispersed by a 1-m normal-incidence monochromator equipped with a 1200 lines/mm grating (blaze wavelength $150 \mathrm{~nm}$ ) and recorded by a position-sensitive CsTe microchannel-plate detector, which is sensitive to wavelengths from 160 to $300 \mathrm{~nm}$. The spectral resolution is better than $30 \mathrm{meV}$. Prior to measurements, the sample inside the absorption cell is outgassed in vacuum at ambient pres-

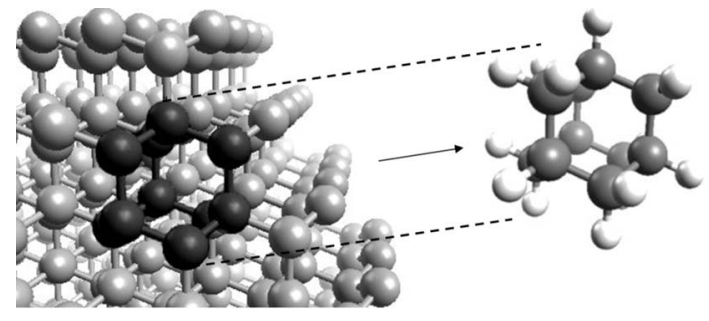

a) Bulk diamond lattice

b) Admantane $\mathrm{C}_{10} \mathrm{H}_{16}$

FIG. 1. Adamantane $\left(\mathrm{C}_{10} \mathrm{H}_{16}\right)$ is the smallest closed-cage diamond nanostructure. It can be constructed by excising a single crystal cell from the bulk diamond lattice (a) and then passivating dangling bonds with hydrogen (b). 

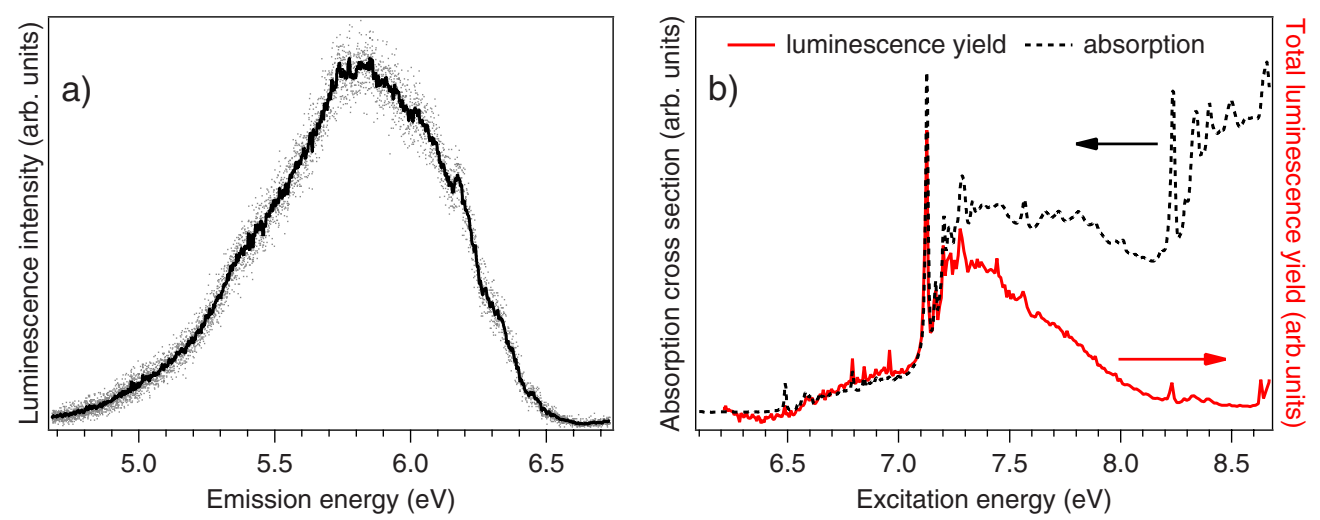

FIG. 2. (Color online) (a) The emission spectrum of adamantane upon UV photoexcitation. (b) The luminescence yield of adamantane compared to its optical absorption (Ref. 8). The differences in absolute intensity at higher energies in panel (b) are due to the different measurement techniques.

sures of $\sim 10^{-5}$ mbar. Measurements are conducted at room temperature $(T=293 \mathrm{~K})$ with an adamantane vapor pressure on the order of 0.1 mbar.

\section{RESULTS AND DISCUSSION}

The spectrally resolved luminescence of adamantane is shown in Fig. 2(a). The luminescence is very broad in energy and ranges approximately from 4.9 to $6.5 \mathrm{eV}$ with a width of more than $0.8 \mathrm{eV}$ (FWHM). Exhibiting a slow increase at the low energy side, the PL reaches its maximum intensity around $5.8 \mathrm{eV}$. The PL intensity drops a little more steeply toward higher energies with no noticeable luminescence beyond $6.5 \mathrm{eV}$. The emission spectrum in Fig. 2(a), recorded at an excitation energy of $7.12 \mathrm{eV}(174.2 \mathrm{~nm})$, is not dependent on the excitation energy. It seems to possess multiple components, which are, however, not all clearly resolvable. In bulk diamond a narrow luminescence band due to intrinsic phonon-assisted recombination has been observed. ${ }^{16}$ The shoulder at $6.2 \mathrm{eV}$ could be due to a similar decay channel including free electron-hole pair recombination over the band edge $(\sim 6.5 \mathrm{eV})$ (Ref. 8$)$ and the excitation of a $\mathrm{CH}$ stretch vibrational mode $(\sim 350 \mathrm{meV}) .{ }^{17}$ The known size and well-defined structure of adamantane, shown in Fig. 1, allow to a priori exclude several effects, which often complicate the interpretation of experimental luminescence spectra, e.g., recombination at defect sites, broadening due to size distribution, or Förster type energy transfer from smaller to larger nanocrystals.

The luminescence of the investigated diamond NC exhibits a considerable red-shift compared to its absorption, displayed in Fig. 2(b). The emission maximum at $\sim 5.8 \mathrm{eV}$ is red-shifted by $0.7 \mathrm{eV}$ with respect to the optical absorption gap of adamantane at $6.49 \mathrm{eV}{ }^{8}$ The difference in absorption and emission energies (Stokes shift) is typically assigned to structural relaxation of the excited state. Experimental results on the size dependence and magnitude of the shift due to such relaxation in $\mathrm{Si} \mathrm{NCs}$ are controversial, with the maximum shift ranging from a few hundred $\mathrm{meV}$ to several eV. ${ }^{18-20}$ Different surface structures and passivants have been predicted to exert strong influence on the Stokes shift in Si
NCs. ${ }^{18}$ For small hydrogenated Si NCs, a shift on the order of $\sim 1 \mathrm{eV}$ is expected due to structural relaxation of the excited state. ${ }^{18,19}$ Nonetheless, one has to consider that diamond nanoparticles possess a more rigid framework than their Si analogs. This rigidity should lead to less structural relaxation upon excitation and thus result in a smaller Stokes shift. Another effect that can lead to large shifts is recombination of self-trapped excitons (STE). STE, which are expected to occur in all types of small semiconductor $\mathrm{NCs},{ }^{21}$ are localized on a single bond and form favorably at the crystallite's surface. Emission from STE states is known to produce shifts on the order of $\sim 1 \mathrm{eV}$ in good agreement with the shift observed. In addition, emission from STE states is typically broad in energy, ${ }^{22}$ just like the emission observed for adamantane. These indicators lead us to believe that the luminescence is due to STE.

To get further insight into the origin of the measured PL, we have recorded a luminescence excitation spectrum of adamantane. The overlay in Fig. 2(b) shows that the measured luminescence intensity scales with the absorption coefficient of adamantane, reproducing all major absorption features. This provides unambiguous evidence that the detected PL originates from the nanodiamond itself. In order to consider the usability of diamond nanostructures for future photonic applications, next, the QY is discussed. A rough estimate gives a PL QY close to $1 \%$. This number has been derived by calculating the number of absorbed photons from the transmission measurements and considering the geometry and the detection sensitivity of the experimental setup. Recent theoretical investigations on similar nanodiamonds predicted radiative lifetimes to be smaller than $\sim 100 \mathrm{~ms}^{23}$ This alone and without reference to nonradiative rates, however, does not allow to draw conclusions about the luminescence QY. As a comparison, we want to discuss the QY in the light of the findings for $\mathrm{Si} \mathrm{NCs}$, the most closely related system. In Si NCs of comparable size luminescence QYs are typically on the order of a few percent when measured at room temperature rising to several ten percent when cooled to liquid nitrogen or even helium temperatures. ${ }^{5}$ Low QYs in Si NCs have been attributed to the coexistence of "bright" and "dark" NCs and $100 \%$ QY is expected for perfect samples. ${ }^{24}$ Adamantane resembles these "bright" NCs because nonradi- 


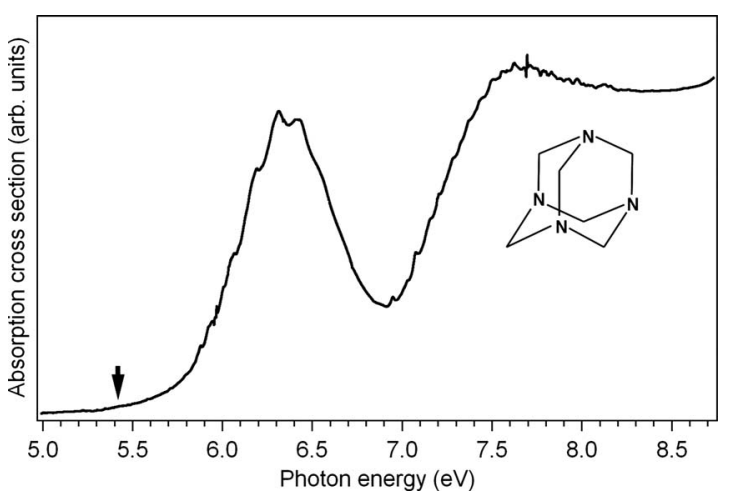

FIG. 3. Optical absorption of urotropine $\left(\mathrm{C}_{6} \mathrm{~N}_{4} \mathrm{H}_{12}\right.$, also hexamethylenetetramine) measured in the gas phase at comparable absorption $(T=390 \mathrm{~K})$. The optical gap at $5.42 \mathrm{eV}$, as defined in Ref. 8, is marked by an arrow.

ative processes due to recombination at structural defects can be excluded. The investigation of the temperature dependence of the observed PL might shed light on the underlying processes and the nature of the competing nonradiative decay channels.

In bulk diamond, typically, nitrogen-vacancy centers are used to introduce visible luminescence. Molecular diamonds with sizes below $1 \mathrm{~nm}$ are too small to incorporate vacancies in their lattice structure. Also, the substitution of atoms on regular bulk diamond lattice sites by single nitrogen atoms will not function as doping, i.e., add free electrons or holes to the host crystal. However, it is important to understand the influence of nitrogen inclusion on the optical properties of nanoscale diamond. As a first step in this direction, we investigated urotropine (hexamethylenetetramine), shown schematically in the structural inset in Fig. 3. The optical absorption spectrum of urotropine is shown in Fig. 3. The spectrum is found to be rather continuous and sharp spectral features, as observed for adamantane, ${ }^{8}$ are absent. The absorption onset is very smooth and the optical gap, as defined in Ref. 8, is determined to be $5.42 \pm 0.04 \mathrm{eV}$, about $1 \mathrm{eV}$ lower than for adamantane. Similar to adamantane, the spec- trum exhibits two broad main bands. The first one ranges from the absorption onset to approx. $7 \mathrm{eV}$ and the second starts at $7 \mathrm{eV}$ and fades into the ionization continuum beyond $8 \mathrm{eV}$. The urotropine structure corresponds to the inclusion of four nitrogen atoms into the adamantane framework meaning a massive "doping" concentration close to unity. Rather than to "doped" adamantane, the additional valence electrons of the $\mathrm{N}$ atoms lead to electron lone-pairs replacing covalently bonded hydrogen. These electron lone-pairs constitute the highest occupied states ${ }^{25}$ which are broad in energy and dominate the optical properties near the gap resulting in a lower optical gap and a smooth appearance of the spectrum. Contrary to adamantane, no UV PL is observed upon excitation between 5.6 and $8.8 \mathrm{eV}$. This lack of UV luminescence suggests that, unlike adamantane, urotropine possesses a structure that strongly favors nonradiative decay.

\section{CONCLUSIONS}

In this work, we have shown that adamantane exhibits very broad intrinsic PL in the UV spectral region. The results suggest that transitions in indirect band gap semiconductors are no longer restricted by momentum conservation rules when dimensions are reduced. The broadness of the emission spectrum raises questions about the precise mechanisms behind the observed luminescence. We propose STE to be responsible for this broadness as well as for the large difference between absorption and emission energies. Similar measurements on urotropine, a highly nitrogen doped diamond cluster, show a strongly deviating optical absorption and no luminescence. The present results show that unreconstructed nanodiamonds, such as diamondoids, are promising candidates for light-emitting devices in the deep UV spectral region.

\section{ACKNOWLEDGMENTS}

This work has been funded by the Deutsche Forschungsgemeinschaft DFG. L.L. is grateful for a scholarship of the Studienstiftung des Deutschen Volkes. Furthermore, the authors would like to thank G. Reichardt (BESSY) for technical support.
${ }^{1}$ T. van Buuren, L. N. Dinh, L. L. Chase, W. J. Siekhaus, and L. J. Terminello, Phys. Rev. Lett. 80, 3803 (1998).

${ }^{2}$ C. Bostedt, T. van Buuren, T. M. Willey, N. Franco, L. J. Terminello, C. Heske, and T. Möller, Appl. Phys. Lett. 84, 4056 (2004).

${ }^{3}$ L. T. Canham, Appl. Phys. Lett. 57, 1046 (1990).

${ }^{4}$ S. Godefroo, M. Hayne, M. Jivanescu, A. Stesmans, M. Zacharias, O. I. Lebedev, G. van Tendelooo, and V. V. Moshchalkov, Nat. Nanotechnol. 3, 174 (2008).

${ }^{5}$ M. Nirmal and L. Brus, Acc. Chem. Res. 32, 407 (1999).

${ }^{6}$ D. Kovalev, H. Heckler, M. Ben-Chorin, G. Polisski, M. Schwartzkopff, and F. Koch, Phys. Rev. Lett. 81, 2803 (1998).

${ }^{7}$ R. C. Fort and P. von R. Schleyer, Chem. Rev. 64, 277 (1964).

${ }^{8}$ L. Landt, K. Klünder, J. E. Dahl, R. M. K. Carlson, T. Möller, and C. Bostedt, Phys. Rev. Lett. 103, 047402 (2009).
${ }^{9}$ J. E. Dahl, S. G. Liu, and R. M. K. Carlson, Science 299, 96 (2003).

${ }^{10}$ T. M. Willey, C. Bostedt, T. van Buuren, J. E. Dahl, S. G. Liu, R. M. K. Carlson, L. J. Terminello, and T. Möller, Phys. Rev. Lett. 95, 113401 (2005).

${ }^{11}$ T. M. Willey, C. Bostedt, T. van Buuren, J. E. Dahl, S. G. Liu, R. M. K. Carlson, R. W. Meulenberg, E. J. Nelson, and L. J. Terminello, Phys. Rev. B 74, 205432 (2006).

${ }^{12}$ K. Lenzke, L. Landt, M. Hoener, H. Thomas, J. E. Dahl, S. G. Liu, R. M. K. Carlson, T. Möller, and C. Bostedt, J. Chem. Phys. 127, 084320 (2007)

${ }^{13}$ T. M. Willey, J. D. Fabbri, J. R. I. Lee, P. R. Schreiner, A. A. Fokin, B. A. Tkachenko, N. A. Fokina, J. E. Dahl, R. M. K. Carlson, A. L. Vance, W. Yang, L. J. Terminello, T. van Buuren, and N. A. Melosh, J. Am. Chem. Soc. 130, 10536 (2008). 
${ }^{14}$ W. L. Yang, J. D. Fabbri, T. M. Willey, J. R. I. Lee, J. E. Dahl, R. M. K. Carlson, P. R. Schreiner, A. A. Fokin, B. A. Tkachenko, N. A. Fokina, W. Meevasana, N. Mannella, K. Tanaka, X. J. Zhou, T. van Buuren, M. A. Kelly, Z. Hussain, N. A. Melosh, and Z.-X. Shen, Science 316, 1460 (2007).

${ }^{15}$ C. Bostedt, T. van Buuren, T. M. Willey, and L. J. Terminello, Appl. Phys. Lett. 85, 5334 (2004).

${ }^{16}$ H. Kawarada, T. Tsutsumi, H. Hirayama, and A. Yamaguchi, Appl. Phys. Lett. 64, 451 (1994).

${ }^{17}$ J. Filik, J. N. Harvey, N. L. Allan, P. W. May, J. E. P. Dahl, S. Liu, and R. M. K. Carlson, Spectrochim. Acta Part A 64, 681 (2006).
${ }^{18}$ A. Puzder, A. J. Williamson, J. C. Grossmann, and G. Galli, J. Am. Chem. Soc. 125, 2786 (2003).

${ }^{19}$ X. Wang, R. Q. Zhang, S. T. Lee, T. A. Niehaus, and T. Fraunheim, Appl. Phys. Lett. 90, 123116 (2007).

${ }^{20}$ M. Lannoo, C. Delerue, and G. Allan, J. Lumin. 70, 170 (1996).

${ }^{21}$ G. Allan, C. Delerue, and M. Lannoo, Phys. Rev. Lett. 76, 2961 (1996).

${ }^{22}$ Y. D. Glinka, S.-H. Lin, L.-P. Hwang, Y.-T. Chen, and N. H. Tolk, Phys. Rev. B 64, 085421 (2001).

${ }^{23}$ M. Nishida, Solid State Commun. 149, 746 (2009).

${ }^{24}$ L. Brus, J. Phys. Chem. 98, 3575 (1994).

${ }^{25}$ W. Schmidt, Tetrahedron 29, 2129 (1973). 